\title{
The cost of providing hypertension health care in public health facilities case study of the NCD clinic at Rwinkwavu district hospital
}

\begin{abstract}
Background: The cost of hypertension healthcare services is increasing worldwide due to increase of its prevalence estimated to $40 \%$ in adults globally and high prevalence $46 \%$ is in Sub-Saharan African. The purpose of this study is to provide information of the cost of hypertension health care services in monetary values on one hand and to determine the cost of health care services of each stage of hypertension at district hospital in rural Rwanda. The study benefits the care givers of hypertension care to understand the socio-economic status of the patients and plan their management.
\end{abstract}

Methods: Retrospective study of 68 patients followed-up in the NCD clinic from January to December, 2013. The data have been collected using patients' files included demographic information, numbers of visits, diagnostic tests and medications. The Ministry of Health tariff, the information from the charts and Excel cost analysis was done to discover the average cost per patient and requirement of good care at each stage of hypertension.

Results: Of the 68 patients analyzed, $74 \%$ were female and $26 \%$ were male. Their socioeconomic status was generally very low; most of the patients had zero formal education $37 \%$ and $34 \%$ patients had only a primary education level; $53 \%$ patients were famers owned small pieces of lands which are not considerably productive and $18 \%$ patients were unemployed. The cost of providing hypertension healthcare services estimated in monetary values was 53,656,736 Rwf excluding the maintenance cost in financial year 2013. The driving cost of hypertension care delivery was the expenditure on medicines, which were estimated at 9,343,956 Rwf per year. The capital cost was $52 \%$ and the recurrent cost was $65 \%$ of the total cost of hypertension care. The average costs of hypertension care per patient for each stage per year were 25,431 Rwf for stage one, 45,812 Rwf for stage two and $82,778 \mathrm{Rwf}$ for stage three.

Conclusion and implications for translation: The overall control of hypertension with insurance coverage is good at Rwinkwavu District Hospital. Hypertension health care services can be effectively implemented in every district hospital in Rwanda through the NCD program. The cost of providing hypertension health care services information are critically missing in the region, more studies are needed on cost of NCDs health care services and their countries' economy impact as the prevalence increasing rapidly.

Keywords: hypertension, out-of-pocket payments, health insurance, ncd, health care services, healthcare access
Volume 9 Issue 2 - 2020

\author{
Deogratias Ndagijimana,' Bethany Hedt \\ Gauthier, ${ }^{2}$ Connie Mureithi, ${ }^{3}$ Alyse Kennedy' \\ 'Partners In Health, Rwanda \\ 2Harvard Medical School, USA \\ ${ }^{3}$ Mount Kenya University, Kenya
}

Correspondence: Deogratias Ndagijimana, MPH, RN, mHealth Mixed Methods Research Coordinator, Partners In Health, Kigali, Rwanda,Tel +250783893064, Email nd.dos09@gmail.com

Received: October 02, 2019 | Published: March 06, 2020

\section{Introduction}

\section{Background of the study}

The cost of health services is rapidly increasing all over the world. There is paucity of cost analysis and unit cost data from the low income-and middle-income countries. The importance of costing information is indispensable as a severe resource constraint affects the health sectors in many countries to day.

In many African countries, lack of access to healthcare affects a large proportion of the population. The financial barriers to health care lead to various forms of exclusion: total exclusion or becoming destitute, seasonal exclusion, temporary exclusion or partial exclusion. The risks of total exclusion from health care or becoming destitute are higher among extremely poor populations. For other segments of the population which depend on weak and irregular incomes, the risks of seasonal, temporary and partial exclusion are higher. ${ }^{2}$

The provision of health services in Rwanda was a state duty, but due to the national economic state of the country; it cannot finance either all or even the majority of the health care out of the general Government budget. This is partly due to increased demand of the quality of services and escalating costs of provision of these services. At the same time out-of-pocket payments have severe efficiency and equity implications. ${ }^{3}$

As the number of hypertension cases worldwide is increasing so are the costs associated with treatment and care. Because the high prevalence of Hypertension is a relatively new phenomenon in 
low-and-middle-income countries there is inadequate information available on what it takes to provide this care specifically in regards to the costs associated with care in resource poor-settings. This information is necessary and without it the health sectors of LMIC are extremely limited in their ability to provide quality and comprehensive hypertension care. ${ }^{1}$

In developing nations, due to socio-economic and infrastructure constraints, access to care, hypertension education and practical protocols are not well established. The difficult reality is also a lack of data supporting the feasibility of adequate and cost-effective treatment and control of hypertension. Most studies on adherence and cardiovascular disease in Sub-Saharan Africa have taken place in urban or mixed settings. ${ }^{4}$

In regards to diagnosing, treating and controlling hypertension in rural areas in Africa the challenges are many and accessibility and affordability are huge barriers. As of 2010 hypertension had begun to emerge across the developing world as the leading cause of death with $80 \%$ of all hypertension deaths according in low and middle-income countries. ${ }^{5}$ Rwanda, like the rest of the developing world, is struggling with the increasing instances of hypertension. Across the country $46 \%$ of all adults in the country suffer from hypertension and 730 hypertension related deaths occur each year. ${ }^{6}$ While the government of Rwanda has acknowledged the problem of hypertension and developed national strategy to address this problem there are still big gaps in the standard of care. This is largely a result of the public's lack of awareness; in certain cross-sectional studies 33\% of hypertension patients were unaware of their status, ${ }^{6}$ access to affordable care and appropriate control of the disease.

When hypertension is not managed properly it creates a huge strain on society. First, unmanaged disease greatly increases the likelihood of severe complications, which require costly and timely interventions. Secondly, hypertensive individuals are limited in their ability to contribute to the socio-economic growth of the country. Given this reality there is a big space for the development of adequate and costeffective interventions for hypertension in Rwanda. Moreover, The Community Based Health Insurance in Rwanda was identified as a privileged channel for the growth of financial accessibility to health services in both rural settings and in the informal sector has increased to the coverage of health care to the population. The Over-utilization of the services by subscribers who hastily solicit health care services, thus have strong impact on the cost of input and output of the management of the hospital.

\section{Aim of the study}

In this paper main objective of research is to provide information of the cost of providing hypertension health care services at Rwinkwavu District Hospital in the NCD clinic.

\section{Specific objectives of the study}

i. To estimate the cost of providing hypertension care services in monetary values in Rwinkwavu District Hospital

ii. To determine the cost of each stage of hypertension health care services per patient over the period of 1 year.

\section{Methods}

The study designed to be retrospective. The study included 68 patient files with hypertension followed at Rwinkwavu District
Hospital in the NCD clinic for the period between January and December, 2013. For the data collection used the paper charts of the patients. The charts are created at intake and filled by clinicians from the NCD clinic at every appointment.

\section{Study variables}

In order to determine the cost of hypertension care I first analyze the demographic data to determine the basic information about the patients and how the cost of hypertension care could affect them.

\section{Statistical analysis}

The information extracted from the paper charts of the patients have entered and analyzed in Excel. The Excel was used to analyze the demographic information; the data analysis has been done on management by stages of hypertension and calculates the cost of hypertension health care services. The descriptive method has been used to quantify the hypertension health care services in monetary values.

\section{Ethical approval}

The proposal was submitted for approval to Mount Kenya University and provided the letter for data collection, then it was submitted to Rwinkwavu Hospital Institutional Review Board (IRB) for review and the approval for research was granted.The study followed the basic ethical principles, maintained confidentiality of private information.

\section{Results}

\section{Sociodemographic characterisitcs}

The analysis found that the most patients followed up in the NCD clinic by 2013 were female. Of the 68 patients analysed 74\% were female and $26 \%$ were male. Generally, over the course of the year females had more appointments than men. This led to better control of hypertension in females' patients compared to men.

Table 1 shows that most patients $72 \%$ lived in Kayonza District but this was not surprising because Rwinkwavu District Hospital is the only hospital with an NCD clinic in Kayonza District.

Table I Catchment status of referring facilities

\begin{tabular}{lll}
\hline Not referred & 15 & $22 \%$ \\
Outside Kayonza catchment & 4 & $6 \%$ \\
Inside Kayonza catchment & 49 & $72 \%$ \\
\hline
\end{tabular}

The analysis of patient's education levels and occupation showed that the socio-economic status was generally very low for the patients sampled. Most of the patients had zero formal education 37\% and $34 \%$ patients had only a primary education level. Finally, there were only $24 \%$ patients with secondary education level and $6 \%$ patients with tertiary education level (Table 2).

Table 2 Education level

\begin{tabular}{lll}
\hline No-school & 25 & $37 \%$ \\
Primary & 23 & $34 \%$ \\
Secondary & 16 & $24 \%$ \\
Tertiary & 4 & $6 \%$ \\
\hline
\end{tabular}


Most of the patients were famers $53 \%$ with small pieces of lands which are not considerably productive and $18 \%$ patients were unemployed. The number of patients employed who were not famers was $21 \%$ and $9 \%$ were self-employed. As most of the patients were famers or unemployed $71 \%$ this means the majority of patients had extremely low or no level of income (Table 3 ).

Table 3 Occupation status

\begin{tabular}{lll}
\hline Employed & 14 & $21 \%$ \\
Unemployed & 12 & $18 \%$ \\
Self-employed & 6 & $9 \%$ \\
Farmer & 36 & $53 \%$ \\
\hline
\end{tabular}

Almost all patients seeking hypertension care 94\% were covered by some type of insurance in line with the policy of the Government of Rwanda that every citizen must have insurance. Having insurance makes the treatment adherence more likely because the patients do not cover all the cost independently. The number of patients covered by Community Based Health Insurance, or Mutuelle, was 79\%, 3\% were covered by MMI. The few patients with employment status, $6 \%$, were covered by Rwanda Social Security Board (RSSB) insurance, and 4\% were covered by SORAS (Med plan). The CBHI plays a main role in health care services accessibility and reduce the burden of cost for health care services (Table 4).

Table 4 Insurance status

\begin{tabular}{lll}
\hline Mutuelle(CBHI) & 54 & $79 \%$ \\
RSSB & 4 & $6 \%$ \\
SORAS & 3 & $4 \%$ \\
MMI & 2 & $3 \%$ \\
Other & 1 & $1 \%$ \\
Unknown & 4 & $6 \%$
\end{tabular}

After looking at the demographic data in order to understand the profile of the patients coming to Rwinkwavu Hospital for hypertension care I looked at the costs associated with the different stages of hypertension. The cost related to each stage is important because the set of costs associated with care is different depending on the stage of hypertension at diagnosis. Most of the patients $60 \%$ presented in the clinic had stage one hypertension, $26 \%$ patients presented had stage two and $13 \%$ presented in stage three.

\section{Main variable results}

The cost related to each stage is important because the set of costs associated with care is different depending on the stage of hypertension at diagnosis. Most of the patients $60 \%$ presented in the clinic had stage one hypertension, $26 \%$ patients presented had stage two and $13 \%$ presented in stage three (Table $5 \& 6$ ).

Table 5 Stage of hypertension

\begin{tabular}{ccc}
\hline Stage I & 40 & $60 \%$ \\
Stage 2 & 18 & $26 \%$ \\
Stage 3 & 9 & $13 \%$ \\
\hline
\end{tabular}

Table 6 Phase of disease at intake

\begin{tabular}{lll}
\hline Chronic & 25 & $37 \%$ \\
Acute(Accelerated) & 36 & $53 \%$ \\
Unknown & 7 & $10 \%$
\end{tabular}

There were two phase of prognosis status. The most patients, 53\%, were in the Acute (Accelerated) phase and $37 \%$ patients were in the chronic phase of hypertension. The costs increase based on number of visits, diagnostic tests exams and drugs. The more advanced the stage the most comprehensive the care becomes and so the cost of hypertension care increases considerably from stage one to stage two and from stage two to stage three. The average number of visits for a patient with stage one hypertension is 4 times per year. For a stage two patient they can be expected to visit the NCD clinic 5 times and for a stage three the number of yearly visits is 6 . During the follow-up in stage 1 they must have creatinine performed once per year, urinalysis 2 times per year and $40 \%$ patients undergo a chest $\mathrm{X}$ ray. The number of diagnostic test and exams per year increases by 2 and 3 times as much for stage two and stage 3 respectively.

The other critical set of costs that needed to by analyses to address the primary objective of the paper, which was to determine in monetary values to the cost of care to Rwinkwavu Hospital. Strong management of hypertension for all three stages of hypertension depends on the availability of medicine, expertise and equipment at the district hospital level. In order to determine what is required to provide adequate care and management of hypertension at all three stages it was necessary to analyse the costs of capital to the clinic over the course of a year.

In Table 7 the total cost related to the clinic's capital was estimated at 18,537,200 Rwf for all of 2013. For this calculation certain costs were excluded such opportunity cost or time and transport. Meal costs to patients and staff as well as general clinic maintenance costs were also excluded. This study also chose not to consider the depreciation in cost for equipment that is currently used in the clinic but over five years old.

Table 8 shows that the total cost of providing hypertension health including costs to the district health center and care services is $35,119,536 \mathrm{Rwf}$ per year. The driving cost of hypertension care delivery is the expenditure on medicines, which is estimated at 9,343,956 Rwf per year. The cost of human resources, such as salary support, should be broken down according to determine what percentage of their salary is associated with hypertension care as compared to other NCD care. The cost of utilities was one on the lower side compared with other recurrent cost elements.

The cost and management plan for hypertension patients depends on the stage of hypertension and the cost increases according to the stage of hypertension. The average cost of hypertension is 40,933 Rwf per patient per year in the NCD clinic. In stage one the average cost of hypertension care is 25,431 Rwf per patient per year, it 2 times of stage one in stage two $(54,812 \mathrm{Rwf})$ and 3 times in stage three $(82,078$ Rwf).

The most used drug was Hydrochlorothiazide at a dose of $25 \mathrm{mg}$ in tablet form $(24 \%)$. The drug is used as a diuretic as a first line treatment of hypertension and is used for all three stages of hypertension. Captopril was responsible for $20 \%$ of overall medication consumption 
and was taken in $25 \mathrm{mg}$ does in tablet form. Captopril is a beta-blocker and used as a third line treatment of hypertension. Amlodipine, given in $10 \mathrm{mg}$ tablets, represented $16 \%$ of overall consumption and belongs in class of calcium channel blockers. This drug is used as a second line treatment of hypertension. Laboratory diagnostic tests are an additional cost and requirement for hypertension care at all three stages. Creatinine tests made up of the bulk of all diagnostic tests at $28 \%, 25 \%$ for Urinalysis and $15 \%$ for Ionogram. Of all patients requiring medical imaging $51 \%$ had Chest X-rays taken, 23\% had Echography performed and $26 \%$ had ECG. Medical imaging was predominantly used for stage three diagnoses.

Table 7 Cost of capital for the NCD clinic at Rwinkwavu hospital for 2013

\begin{tabular}{|c|c|c|c|c|c|}
\hline Designation & Specification & Qty & U.P & Total cost & Annual cost \\
\hline Building & & 1 & $4,200,000$ & $4,200,000$ & 140,000 \\
\hline \multirow[t]{5}{*}{ Assets } & Clinical exam table & 1 & 85,000 & 85,000 & 17,000 \\
\hline & Table & 1 & 45,000 & 45,000 & 4,500 \\
\hline & Chairs & 3 & 15,000 & 45,000 & 4,500 \\
\hline & Cabinet & 2 & 85,000 & 170,000 & 17,000 \\
\hline & Multi-socket & 2 & 5,000 & 10,000 & 3,333 \\
\hline \multirow[t]{10}{*}{ Equipment } & Ultrasound machine & 1 & $15,000,000$ & $15,000,000$ & $\mathrm{I}, 500,000$ \\
\hline & ECG machine & 1 & $20,500,000$ & $20,500,000$ & $2,050,000$ \\
\hline & X-ray machine & 1 & $80,000,000$ & $80,000,000$ & $2,666,667$ \\
\hline & PB machine & 2 & 18,000 & 36,000 & 18,000 \\
\hline & Negatoscope & 1 & 50,000 & 50,000 & 10,000 \\
\hline & Oeutoscope & 2 & 35,000 & 70,000 & 14,000 \\
\hline & Glucometer & 2 & 98,000 & 196,000 & 39,200 \\
\hline & Oxymeter & 2 & 85,000 & 170,000 & 34,000 \\
\hline & Weight scale & 1 & 20,000 & 20,000 & 4,000 \\
\hline & Dustbin & 2 & 15,000 & 30,000 & 15,000 \\
\hline Car & Toyota land cruiser & 1 & $60,000,000$ & $60,000,000$ & $12,000,000$ \\
\hline Total & & & & $180,627,000$ & $18,537,200$ \\
\hline
\end{tabular}

Table 8 Recurrent costs related to hypertension care at the NCD clinic of Rwinkwavu hospital

\begin{tabular}{llll}
\hline Designation & Specification & Cost per month & Total cost per year \\
\hline Medicines & Hypertension drugs & 778,663 & $9,343,956$ \\
Utilities & Electricity & 10,000 & 120,000 \\
& Water & 5,000 & 60,000 \\
Human resources & Doctors(2) & 703,286 & $8,439,432$ \\
& Nurses(3) & 636,429 & $7,637,148$ \\
& MER Officer(I) & 305,680 & $3,668,160$ \\
& Driver(I) & 74,250 & 891,000 \\
Vehicles & Cleaner(I) & 45,320 & 543,840 \\
Stationary & Fuel & 33,000 & 396,000 \\
Social support & & 15,000 & 180,000 \\
Total & & 320,000 & $3,840,000$ \\
\hline
\end{tabular}


The completed data analysis uncovered the comprehensive cost of providing hypertension health care services. The services that contributed to the overall cost of hypertension care at the NCD Clinic at Rwinkwavu Hospital were mainly medications, diagnostics test and other form of treatment related costs as well as the capital cost and human resources to run the clinic. It was found that the stage three of Hypertension was costlier than stage one and stage two. While that of capital cost despite some missing components such as maintenance cost, it was realized that it is less costly comparing to the recurrent cost.

\section{Discussion}

Globally the literature on hypertension says that hypertension prevalence is estimated $40 \%$ in $\geq 25$-year-old adults, Sub-Saharan Africa had the highest rate at $46 \%$ and it is increasing worldwide it is projected to be the number one cause of death globally by $2020 .{ }^{8}$ The death from hypertension was 7.5 millions $2008 .{ }^{8}$ There are few studies that talks about the cost of providing hypertension care in developed world and developing world; in both cases the high cost is a problem and for Low and middle income countries presently face the greatest economic burden. The hypertension expenditures have a large burden to the economy in USA $12.6 \%$ of national spend on allocation of hypertension health care. In American population the prescriptions of hypertension play a big role in increasing cost; this study found that only $40 \%$ of prescriptions of hypertension were evidence based. The evidence-based prescription may help for annual provision and reduction of cost of hypertensive drugs. The adherence on evidencebased prescription may significantly increase the prescription saving to $42 \%$ of program cost. ${ }^{10}$ There are currently two primary guidelines for hypertension treatment that are used internationally. The first approach is to base treatment on individuals having a certain number of risk factors that have a certain level of severity. The second approach defines and treats hypertension using a certain level of blood pressure. ${ }^{11}$ These international guidelines however, are not specific to Africa and do not take into account the very many socio-economic and geographic challenges that make diagnosis, treatment and control easy. They also do not acknowledge the cost of giving care based on these protocols in Sub-Saharan Africa, which will be different given many additional economic, geographic, social and political challenges that come with providing care.

The literature in Sub-Saharan Africa says that the prevalence of hypertension is high both urban and rural area with low levels of detection, treatment and control indicating the need for costeffective strategies for primary prevention, detection and treatment of hypertension and the growing public health challenge of noncommunicable diseases in Sub-Saharan Africa. ${ }^{12}$ The cost of hypertension care may be a Barrie for hypertension control because the costs of hypertension care press a major challenge in African countries. ${ }^{13}$ Lack of access to primary health care of hypertension lead to many cases of uncontrolled hypertension combined with other risk factors like low level of education and low socio-economic status. ${ }^{14}$ In Kenya the individual needs to buy the drugs in $23 \$$ a month. ${ }^{15}$

The information on cost of hypertension health care services critically missing in the region and there is no considerable research done on that. In South Africa the implementation of prescribing anti-hypertensive drugs at Community Health Center decreased the cost of hypertension care to $24.2 \%$. This is attributable to reduce the prescription of very expensive drugs. ${ }^{16}$
For Rwanda there is no information available on the cost of providing hypertension health care services and its economic impact has not been rigorously studied. Cost-effective medicines to treat noncommunicable diseases including hypertension are available although they remain inaccessible and unaffordable to many who need them especially in low and middle income countries where the prevalence of non-communicable diseases are increasing especially cardiovascular diseases. However, challenges to scaling up access to NCD medicines reflect each country's situation and need to be addressed in a country specific way to achieve sustainable and equitable accessibility. The lack of information on cost of providing hypertension health care services remain a big challenge to health settings institutions because it is difficult for the management of the hospital to achieve its objectives, if the culture on costing of health services is not initiated in the public hospitals. The shortage of funds would result in poor quality services for the catchments population and can even cause the stock out of medicines, supplies and poor maintenance of equipment, buildings and others. The problem of accessing essential drugs remains acute due to the low purchasing power of the population and weak pricing regulatory system. ${ }^{7}$

Once the cost data are readily available on cost of providing hypertension health care services, such as coverage of the target population the useful assessments of efficiency in an input/output sense can be made for the rational and equitable use of available resources at least preliminary judgments which will help on fairness of the health scheme1. There may be a difference of mismatching between budget and expenditures if that budget is poorly prepared this may express the miss understanding of required resources to achieve the provision objective of hypertension health care services. This may also be inadequate allocation of resources.

After reviewing the literature I have realized the lack of providing hypertension health care services are and generally the cost of health care services is not rigorously studied in the region.

\section{Critical review}

After reviewing the literature It have been realized that there is a lack of information on cost of providing hypertension health care services are and generally the cost of health care services is not rigorously studied in the region. The data on costing generally are critically missing in the region. For the management of the hospital, there is an information gap between the actual hospital costs and budget provision of quality health services. The knowledge on costing is a necessity for the sustainable and successful implementation of the health insurance policy in Rwanda. It is difficult for the management of the hospital to achieve its objectives, if the culture on costing of health services is not initiated the hospital. The researchers and health institution should do more research on costing of health care services for better planning of different programs in term of better allocation of resources in equitable manner. The studies should focus more on rational use of resources and cost-effective analysis of health care services. It is very important for the health economists to know the cost of health care services; resources need to run health care programs and its impacts on quality of health.

\section{Limitations}

This study will include all patients diagnosed with hypertension at the NCD clinic at Rwinkwavu District Hospital who are consequently followed over the course of a year beginning from January 2013 and 
ending in December, 2013. The study can be generalized to those individuals who present with hypertension at the district hospital level throughout Rwanda.

\section{Recommendation for further studies}

The policy makers should recognized and support diagnosis, rational use of drugs, monitoring of response to treatment, epidemiological surveillance, and research activities.

\section{Conclusion and implications for translation}

From the findings of the study, the following conclusions could be drawn: The cost of providing hypertension health care services in Rwinkwavu District Hospital in the financial year 2013 amounted to $53,656,736$ Rwandan francs. The recurrent cost budget was $35,119,536$ by that constituting $65 \%$ of the total cost. Whereas the capital cost constituted itself 18,537,200 Rwf, excluding maintenance cost. It was also found that the cost of providing hypertension health care services for stage three of hypertension is highly compared to the remaining stages. Within the CBHI the hypertension health care services are accessible which led to the adherence on treatment and help in management and control of hypertension. The long-term follow-up of hypertensive patients is comprehensive at Rwinkwavu Hospital in the NCD Clinic. Hypertension health care services can be effectively implemented in every district hospital in Rwanda through the NCD program.

\section{Key message}

a. The costing information of providing health care services is very necessary to indicate the amount of fund likely to be required to run the sustainable programs.

b. It is important that Ministry of Health improve the quality of the NCD health care services in support of quality health care delivery at all levels.

c. The policy makers should recognized and support diagnosis, rational use of drugs, monitoring of response to treatment, epidemiological surve

d. illance, and research activities.

\section{Ethics approval}

The letter for data collection obtained from Mount Kenya University and obtained authorization of research from Rwinkwavu District Hospital.

\section{Acknowledgments}

Mount Kenya University Administration, all lecturers from nursing school for their knowledge and skills that have been core element for guidance to achieve this level. Special recognization for the family of Mr. Muhayimana Edison \& Mrs. Kayitesi Claudine for their invaluable support.

\section{Conflicts of interest}

The authors declare that there was no conflict of interest.

\section{Funding}

There was no funding/support for this study.

\section{References}

1. Andrew C, David P. Cost Analysis in Primary Health Care. World Health Organization. 1994.

2. Rwanda Ministry of Health. Rwada Community Based Health Insurance Policy. 2010

3. De Brouwere V, Richard F, Witter S. Access to maternal and perinata health services: Lessons from successful and less successful examples of improving access to safe delivery and care of the newborn. Trop Med Int Heal. 2010;15(8):901-909.

4. Labhardt ND, Balo JR, Ndam M, et al. Improved retention rates with low-cost interventions in hypertension and diabetes management in a rural African environment of nurse-led care: a cluster-randomised trial. Trop Med Int Health. 2011;16(10):1276-1284.

5. Twagirumukiza M, Van Bortel LM. Management of hypertension at the community level in sub-Saharan Africa (SSA): towards a rational use of available resources. J Hum Hypertens. 2011;25(1):47-56.

6. Banyangiriki J, Phillips J. Prevalence of Hypertension among Working Adults in Rwanda. Iran J Publ Heal. 2013;42(8):925-926.

7. Rwanda Ministry of Health. Rwanda National Medical Laboratory Policy. 2005.

8. Peer N. Hypertension: now is the time for action. South Africa Med Res Counc. 2013;(5):5-8

9. Hodgson TA, Cai L. Medical Care Expenditures for Hypertension , Its Complications, and Its Comorbidities. Med Care. 2001;39(6):599-615.

10. Fischer MA, Avorn J. Economic Implications of Evidence-Based Prescribing for Hypertension Can Better Care Cost Less?. JAMA. 2004;291(15):1850-1856.

11. Gaziano T, Steyn K, Cohen DJ, et al. Cost-effectiveness analysis of hypertension guidelines in South Africa: absolute risk versus blood pressure level. Circulation. 2005;112(23):3569-3576.

12. Edwards R, Unwin N, Mugusi F, et al. Hypertension prevalence and care in an urban and rural area of Tanzania. J Hypertens. 2000;18(2):145-152.

13. Shulman NB, Martinez B, Brogan D, et al. Financial Cost as an Obstacle to Hypertension Therapy. Am J Public Health. 1986;76(9):1105-1108.

14. Patrick DL. Hypertension Control. Am J Public Health. 1994;84(11):10 12.

15. The East African. Reducing cost of drugs for diabetes, hypertension.

16. Edwards PR, Lunt DW, Fehrsen GS, et al. Improving cost- effectiveness of hypertension management at a community health centre. S Afr Med J. 1998;88(5):549-554 